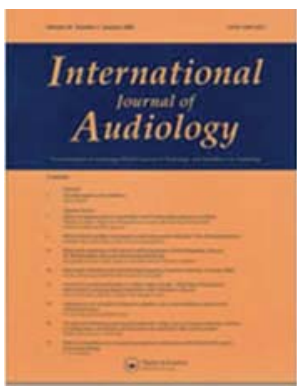

\title{
Process evaluation of Internet-based cognitive behavioural therapy for adults with tinnitus in the context of a randomised control trial
}

\begin{tabular}{|c|c|}
\hline Journal: & International Journal of Audiology \\
\hline Manuscript ID & TIJA-2016-12-0373.R1 \\
\hline Manuscript Type: & Original Paper \\
\hline Date Submitted by the Author: & $\mathrm{n} / \mathrm{a}$ \\
\hline Complete List of Authors: & $\begin{array}{l}\text { Beukes, Eldre; Anglia Ruskin University, Department of Vision and Hearing } \\
\text { Sciences } \\
\text { Manchaiah, Vinaya; Lamar University, Department of Speech and Hearing } \\
\text { Science; The Swedish Institute for Disability Research, Department of } \\
\text { Behavioural Sciences and Learning; Audiology India, Speech and Hearing } \\
\text { Baguley, David; National Institute for Health Research, Nottingham } \\
\text { Hearing Biomedical Research ; Anglia Ruskin University, Department of } \\
\text { Vision and Hearing Sciences } \\
\text { Allen, Peter; Anglia Ruskin University, Department of Vision and Hearing } \\
\text { Sciences; Vision and Eye Research Unit, Anglia Ruskin University } \\
\text { Andersson, Gerhard; Linkopings universitet, 8. Department of Behavioral } \\
\text { Sciences and Learning; Psychiatry Section, Karolinska Institutet, } \\
\text { Department of Clinical Neuroscience }\end{array}$ \\
\hline Keywords: & $\begin{array}{l}\text { Tinnitus, Tele-audiology/Tele-health, Conditions/Pathology/Disorders, } \\
\text { Assistive Technology }\end{array}$ \\
\hline \multicolumn{2}{|c|}{$\begin{array}{l}\text { Note: The following files were submitted by the author for peer review, but cannot be converted to } \\
\text { PDF. You must view these files (e.g. movies) online. }\end{array}$} \\
\hline Figure 1 process eval.pdn & \\
\hline
\end{tabular}

\section{SCHOLARONE \\ Manuscripts}




\section{Process evaluation of Internet-based cognitive behavioural therapy for adults with tinnitus in the context of a randomised control trial}

Eldré W. Beukes, ${ }^{1}$ Vinaya Manchaiah, ${ }^{2,3,4,5}$ David M. Baguley, ${ }^{1,6,7}$ Peter M. Allen, ${ }^{1,8}$ and Gerhard Andersson ${ }^{9,10}$

1. Department of Vision and Hearing Sciences, Anglia Ruskin University, Cambridge, United Kingdom

2. Department of Speech and Hearing Sciences, Lamar University, Beaumont, Texas, USA

3. Linnaeus Centre HEAD, Swedish Institute for Disability Research, Department of Behavioral Science and Learning, Linköping University, Linköping, Sweden

4. Audiology India, Mysore, Karnataka, India

5. Department of Speech and Hearing, School of Allied Health Sciences, Manipal University, Karnataka, India

6. National Institute for Health Research [NIHR], Nottingham Biomedical Research Centre, Ropewalk House, 113 The Ropewalk, Nottingham, United Kingdom

7. Otology and Hearing Group, Division of Clinical Neuroscience, School of Medicine, University of Nottingham, Nottingham, United Kingdom

8. Vision and Eye Research Unit, Anglia Ruskin University, Cambridge, United Kingdom

9. Department of Behavioral Sciences and Learning, Linköping University, Linköping, Sweden

10. Department of Clinical Neuroscience, Division of Psychiatry, Karolinska Institute, Stockholm, Sweden 


\section{Address for correspondence:}

Eldré W. Beukes, Department of Vision and Hearing Sciences, Faculty of Science and Technology, Anglia Ruskin University, Cambridge CB1 1PT, UK. E-mail:

eldre.beukes@anglia.ac.uk 


\author{
Keywords \\ Process evaluation, Satisfaction, Clinical trial, Tinnitus, Tinnitus treatment, Internet- \\ intervention, Cognitive behavioural therapy
}


Beukes. Process evaluation implementing iCBT for tinnitus in a RCT

\section{Process evaluation of Internet-based cognitive behavioural therapy for adults with tinnitus in the context of a randomised control trial}

Eldré W. Beukes, ${ }^{1}$ Vinaya Manchaiah, ${ }^{2,3,4,5}$ David M. Baguley, ${ }^{1,6,7}$ Peter M. Allen, ${ }^{1,8}$ and Gerhard Andersson ${ }^{9,10}$

1. Department of Vision and Hearing Sciences, Anglia Ruskin University, Cambridge, United Kingdom

2. Department of Speech and Hearing Sciences, Lamar University, Beaumont, Texas, USA

3. Linnaeus Centre HEAD, Swedish Institute for Disability Research, Department of Behavioral Science and Learning, Linköping University, Linköping, Sweden

4. Audiology India, Mysore, Karnataka, India

5. Department of Speech and Hearing, School of Allied Health Sciences, Manipal University, Karnataka, India

6. National Institute for Health Research [NIHR], Nottingham Biomedical Research Centre, Ropewalk House, 113 The Ropewalk, Nottingham, United Kingdom

7. Otology and Hearing Group, Division of Clinical Neuroscience, School of Medicine, University of Nottingham, Nottingham, United Kingdom

8. Vision and Eye Research Unit, Anglia Ruskin University, Cambridge, United Kingdom

9. Department of Behavioral Sciences and Learning, Linköping University, Linköping, Sweden

10. Department of Clinical Neuroscience, Division of Psychiatry, Karolinska Institute, Stockholm, Sweden 


\section{Address for correspondence:}

Eldré W. Beukes, Department of Vision and Hearing Sciences, Faculty of Science and Technology, Anglia Ruskin University, Cambridge CB1 1PT, UK. E-mail:

eldre.beukes@anglia.ac.uk 


\begin{abstract}
Objective: The research objective was to identify processes that could either facilitate or hinder clinical implementation of an Internet-based cognitive behavioural therapy intervention for tinnitus in the UK. This was done by exploring the research context, the intervention components and the factors that contributed to the outcomes obtained.

Design: This study investigated eight processes including the recruitment strategies, reach, research context, treatment dose delivered and received, implementation fidelity, barriers to implementation and effectiveness of the intervention.
\end{abstract}

Study sample: Of the 169 registered participants, 146 were randomly assigned to the experimental or control groups ( 23 were excluded). The mean age was 55.57 years with an average tinnitus duration of 11.63 years.

Results: The intended sample of people with distressing tinnitus who were underserved with evidence-based tinnitus interventions was reached. The full guided intervention was delivered. The recommended modules were read more than the optional modules. Intervention components such as the easily readable format and the benefits of the applied relaxation programme facilitated significant positive post-intervention outcomes. Barriers hampering the intervention application included time pressures and low self-motivation.

Conclusions: Results of this process evaluation together with the outcome data can be used to facilitate translating this research into clinical practice.

\title{
Keywords
}

Process evaluation, Satisfaction, Clinical trial, Tinnitus, Tinnitus treatment, Internetintervention, Cognitive behavioural therapy 


\section{Abbreviations}

GAD-7: The Generalised Anxiety Disorder

HHIA-S: Hearing Handicap Inventory for Adults-Screening version

iCBT: Internet-based cognitive behavioural therapy for tinnitus (iCBT)

ISI: Insomnia severity index

PHQ-9: Patient Health Questionnaire

RCT: Randomised control trial

SD: Standard deviation

TFI: Tinnitus Functional Index

THI-S: Tinnitus Handicap Inventory - Screening version

UK: United Kingdom

WCI: Weekly check-in control group 
Beukes. Process evaluation implementing iCBT for tinnitus in a RCT

\section{Introduction}

Providing effective prevention campaigns and treatment routes is at the heart of healthcare (Wanless, 2004). The appropriate level of care is not always achieved due to various barriers preventing delivery of appropriate interventions (Sung et al., 2003; Grol, 2001). One such barrier is the poor translation of health-related research into clinical practices (Grol, 2001). This can lead to discrepancies in evidence-based practice and to the public failing to benefit optimally from advances in health care (Grimshaw et al., 2012). These discrepancies can be minimised by ensuring new interventions are appropriately conducted, evaluated and disseminated to both the scientific and professional community. Translation can also be optimised by identifying factors that can either facilitate or hamper the application of any new intervention (Saunders et al., 2005). Process evaluation models can provide such a framework for evaluating an intervention's potential (Manchaiah et al., 2014).

Process evaluation models for public health interventions and research have gradually developed since the 1960's and have become more complex (Steckler et al., 2002). These models provide a framework for organising conceptual thinking about key process components. The three models that have been widely used in healthcare interventions are the Reach, Efficacy, Adoption, Implementation and Maintenance framework (RE-AIM; Glasgow et al., 1999; Dzewaltowski et al., 2004) and the components suggested by Baranowski and Stables (2000) and Linnan and Steckler (2002). Although each model is unique, there is some overlap. The RE-AIM model includes five dimensions namely: (i) reach, investigating the extent to which the intervention was received by the targeted group; (ii) effectiveness, related to the impact of the intervention as measured by the selected outcome measures; (iii) adoption, associated with delivering the intervention; (iv) implementation, investigating whether the protocol was followed as planned and looks at the delivery of the intervention; 
Beukes. Process evaluation implementing iCBT for tinnitus in a RCT

and (v) maintenance, related to the degree the intervention and results and involvement can be sustained over time. The 11 components suggested by Baranowski and Stables (2000) covered: (i) recruitment procedures; (ii) maintenance; (iii) the specific context; (iv) resources required; (v) implementation of the programme; (vi) reach; (vii) barriers encountered; (viii) exposure to the intervention; (ix) initial use; (x) continued use; and (xi) contamination related to the extent to which additional treatments were received. Linnan and Steckler (2002) suggested inclusion of seven components namely: (i) context; (ii) reach; (iii) dose delivered related to the intended intervention components to be provided; (iv) dose received indicating engagement with the intervention on an individual level; (v) fidelity investigating the extent to which the intervention was delivered as planned; (vi) implementation; and (vii) recruitment.

Process evaluation is particularly important for complex interventions, often required for chronic conditions, which place substantial strain on health-care systems worldwide (Moore et al., 2015). One such chronic condition is tinnitus, which is highly prevalent, with figures found between $10-15 \%$ in the UK (Davis \& Rafaie 2000). There are concerns that the prevalence of tinnitus is on the rise secondary to an increase in noise and music exposure, which are risk factors for developing tinnitus (Martinez et al., 2015). Although there are various recommended treatment approaches for tinnitus including patient education, sound therapy and the use of hearing aids, research supporting the efficacy of many of these interventions is hampered by poor methodologies (Landgrebe et al., 2012). The intervention with the most evidence at present for efficacy in reducing tinnitus distress is cognitive behavioural therapy (CBT; Hesser et al., 2011; Grewal et al., 2014). Despite this evidence, there is limited provision of CBT in clinical practice, largely due to a shortage of trained professionals to provide CBT for tinnitus (Gander et al., 2011). To increase access to CBT for 
tinnitus, a guided Internet-based intervention (iCBT) for tinnitus was developed for the UK (Beukes et al., 2016), following the model used in Sweden (Andersson et al., 2002), and Germany (Weise et al., 2016). Unique to this intervention was that it was guided by an Audiologist, whereas Clinical Psychologists have guided previous interventions. It was then tested for feasibility and piloted (Beukes et al., 2017a). A randomised control trial (RCT) indicated the efficacy of $\mathrm{iCBT}$ for both tinnitus distress and some associated problems such as insomnia, depression, hyperacusis, cognitive failures and quality of life. These effects were maintained two months post-intervention for the experimental group (Beukes et al., 2017b). Further evaluations are underway comparing the iCBT treatment to that of standard clinical care (Beukes et al. 2017c). If this intervention is indeed to be implemented as a credible intervention, factors facilitating and hampering its application need to be identified (Saunders et al., 2005). Determining how much iCBT treatment is required for positive change is for instance still unknown. Integrating information obtained from outcome data and process evaluation can optimise dissemination of new interventions (Moore et al., 2015).

Despite the relevance of process evaluation to evaluate new interventions, the only comprehensive process evaluation to date in the field of Audiology is that of Gussenhoven et al. (2015). A vocational enablement protocol was evaluated for employees with hearing difficulties in clinical practice. They used the framework outlined by Linnan and Steckler (2002) and this research drew inspiration from their study. The research objective of the present study was to identify processes that could facilitate or hinder clinical implementation of an Internet-based cognitive behavioural therapy intervention for tinnitus in the UK. This was done by exploring the:

1. Research context and how this affected the intervention coverage 
Beukes. Process evaluation implementing iCBT for tinnitus in a RCT

2. Delivery of the intervention components and how participants engaged with these

3. Factors that contributed to the outcomes obtained

\begin{abstract}
Method
Study design

Process evaluation was run parallel to a randomised control trial, with a two-month follow-up to evaluate the efficacy of iCBT (Beukes et al., 2015). The experimental group received treatment for 8 weeks, while the control group were monitored weekly by means of the Tinnitus Handicap Inventory - Screening Version (THI-S; Newman, Sandridge \& Bolek, 2008). Once the experimental group had completed treatment, the control group underwent the same intervention. This allowed for intervention effects to be evaluated between and within groups at two different time points for two independent groups.
\end{abstract}

\title{
Ethical considerations
}

Ethical approval was granted by the Faculty Research Ethics Panel of Anglia Ruskin University (FST/FREP/14/478) and the study was registered with Clinical Trials.gov: NCT02370810, date 05/03/2015. It was conducted in accordance with good clinical practice together with the ethical principles of the Declaration of Helsinki. A protocol was used to ensure the security of participants' confidentiality when using the web-portal, complying with the following UK legislation: The Data Protection Act of 1998 and The Privacy and Electronic Communications (EC Directive) Regulations (Riach, 2003).

\section{Participants}

There were 244 participants who registered interest in undertaking this intervention in an RCT trial format on the study website (www.tacklingtinntius.co.uk). Of those 169 (69.26\%) 
completed the initial screening questionnaire. Eligibility for the study was determined in a two-stage process. Initially, participants completed the baseline measurements online. Following completion, a telephonic screening was arranged, to ensure participants fulfilled the study requirements. Inclusion criteria included being aged 18 or over and living in the UK. Computer and Internet access were required. Additional criteria included a minimum tinnitus duration of three months and score of 25 or above on the Tinnitus Functional Index (TFI; Meikle et al., 2012). The exclusion criteria were reporting any major medical, psychiatric or mental disorder and having tinnitus as a consequence of a medical disorder still under investigation.

Of those who completed the screening questionnaire, 23 were excluded, mainly due to tinnitus severity scores being below that indicating the necessity for a clinical intervention. The remaining 146 were stratified for tinnitus severity and age and randomly assigned to the experimental $(n=73)$ and the control groups $(n=73)$ as seen in Figure 1.

\section{[Figure 1 near here]}

\section{The Intervention}

The iCBT intervention incorporated CBT principles, as these presently have the most robust evidence of effectiveness in minimising the effects of tinnitus (Hesser et al., 2011). The programme originally designed by Andersson et al. (2002) and later updated by Andersson and Kaldo-Sandström, (2003) and Kaldo et al. (2007), was selected due to its strong theoretical base. It was translated into English for a study in Australia (Abbott et al., 2009). It was later developed specifically for a UK population by Beukes et al. (2016) to include interactive elements such as quizzes, videos, diagrams and to accommodate different learning styles. It ran over an 8-week period and covered a broad and comprehensive spectrum, 
consisting of 21 modules, of which five were optional as outlined in Table 1. Individual modules were organised into a clear structure, including an overview, explanation and rational, step-by-step instructions and further help section, covering possible difficulties that may have been experienced with each of the techniques suggested.

\section{Process evaluation parameters}

The parameters used were selected from the three models widely applied to intervention delivery, namely the RE-AIM model (Glasgow et al., 1999), those by Baranowski \& Stables, (2000) and the framework by Linnan and Steckler (2002). The processes selected covered a broad spectrum and addressed the specific research objectives of this study. Data collection was multifaceted and consisted of different measures at different time points as outlined below:

\section{Processes related to the research context included:}

Recruitment: this process identified the procedures used to approach and attract participants to participate in the study. It investigated the effectiveness of these recruitment strategies in creating awareness of the trial in the targeted population. Google analytics was used to evaluate the recruitment processes by monitoring the traffic on the recruitment website and to examine trends related to who was interested in the intervention.

Reach: investigated the extent to which the intervention was received by the targeted population. The target population consisted of those with distressing tinnitus who were underserved with evidence-based interventions. A demographic questionnaire was used to determine the demographical profile of the participants and included questions regarding 
tinnitus duration, previous medical examinations related to tinnitus and previous tinnitus treatments.

Context: was connected to the social, demographical and socio-economic characteristic of the participants that may affect generalisability of the outcomes

The following self-reported questionnaires were used to determine the research context:

1) The TFI consisting of 25 items scored on a scale of 0-100 (Meikle et al. 2012) was selected to measure tinnitus distress.

2) The Insomnia Severity Index comprising of seven questions, scored between 0-28 (ISI; Bastien et al. 2001) was used to determine the presence of insomnia

3) The Generalised Anxiety Disorder made up of seven items, scored between 0-21 (GAD-7; Spitzer et al. 2006) was selected to quantify the level of anxiety.

4) The Patient Health Questionnaire (PHQ-9; Spitzer et al. 1999) was chosen to establish the presence of depression. The nine items are scored between 0-28.

5) The Hearing Handicap Inventory for Adults-Screening version (HHIA-S; Newman et al. 1991) was also administered to assess difficulty hearing. The 10 items are scored between $0-40$.

6) Commitment and motivation ratings were used to rate how committed and motivated participants were to the programme on a scale of 1-10 at the start of the intervention with " 1 ” representing not committed and not motivated and ' 10 ' representing very committed and very motivated.

E-mail: editor-ija@utdallas.edu URL: http://mc.manuscriptcentral.com/tija 
Processes related to the intervention delivery involved:

Treatment (dose) delivered: this was defined as intervention components actually provided to participants. This encompassed the content of the intervention, the nature of the guidedintervention and how this may influence outcomes. This was determined by investigating the modules provided, the number of messages sent and the influence of the characteristics of the health professional guiding the intervention.

Treatment (dose) received: this referred to the extent to which participant's engaged and adhered to the intervention. It was determined by data logging from the intervention website. Logs of system usage were kept, such as the number of times participants accessed the intervention, which modules were read, and how many worksheets they completed. The number of messages sent by participants was also recorded. The Tinnitus Handicap Inventory - Screening version (THI-S; Newman et al, 2008) was used to compare dose received with changes in tinnitus distress over time.

\section{Processes related to the outcomes obtained were:}

Implementation fidelity: this was defined as the degree to which the protocol was carried out as intended. As intervention fidelity can be a moderator of the relationship between interventions and their intended outcomes, it is important to investigate (Dusenbury et al., 2003). It was determined by comparing the actual protocol to the intended protocol described in Beukes et al. (2015). 
Beukes. Process evaluation implementing iCBT for tinnitus in a RCT

Barriers to implementation: this process investigated barriers that may affect the implementation of iCBT.

A post-intervention satisfaction questionnaire designed for this particular intervention was used to identify barriers regarding the suitability, content, usability, presentation, and exercises on the intervention. The questionnaire consisted of 15 questions. A five-point Likert-scale scale was used with " 1 " representing strongly disagree and "5" representing strongly agree. This was completed by each group after they completed the intervention. Open-ended questions were also used to offer the opportunity for participants to add any positive or negative comments and any suggestions regarding the iCBT intervention.

Facilitation of effectiveness: this process identified which aspects impacted on intervention effectiveness from the participant's perspective. The information obtained was related back to the context of the outcome data.

To examine effectiveness, a benefit questionnaire was used to rate the iCBT modules. The questionnaire consisted of a five-point Likert-scale, with " 1 " representing not useful and "5" representing very useful.

\section{Data Analysis}

The Statistical Package for Social Sciences version 23.0 was used for data analyses.

Descriptive statistics were used to evaluate the sample characteristics and ratings provided. For comparison purposes, individual scores for each assessment measure were converted to percentages. Baseline group differences were analysed using independent sampled $t$-tests for continuous variables and Chi-Squared tests for categorical variables. 
Beukes. Process evaluation implementing iCBT for tinnitus in a RCT

\section{Results}

\section{Processes related to the research context included:}

Recruitment processes

A comprehensive study website was designed for recruitment purposes. It included sections detailing the intervention, what happens during the study, who the study is suitable for, and contact details if more information was required. There was a link on the website to register for the study. Those registered were invited to complete the screening questionnaire after the study commenced. To improve coverage, a press release was written which gave information about tinnitus, the study and how to register. It was published in newspapers, and magazines across the UK. To target those who use the Internet, Twitter, forums, Facebook and websites containing information about clinical trials were used to share the study information.

Google Analytics indicated that there were around 2,300 sessions from 1,003 users on the recruitment website during the recruitment period. The majority of the views were from the UK. There were views from other countries, possibly linked to the trial being registered on clinicaltrials.gov and therefore easily located worldwide. The most views were on the day that recruitment went live with 908 page views. The average session duration was 10 minutes, indicating that those interested thoroughly considered the information presented regarding the trial. The majority of the visitors $(59.76 \%)$ returned to the recruitment pages. The main locations of those visiting the website were London, Southampton, Manchester, Hastings, and Birmingham. The main pages searched on the recruitment website were 'who may join,' 'about the programme' and 'what happens during the study.' The recruitment strategies were successful at creating enough interest in the trial. The required number of participants were recruited according to sample size calculations for this particular trial. 
Beukes. Process evaluation implementing iCBT for tinnitus in a RCT

Reach

Participants were spread across the UK, although the majority were based in England, as seen in Figure 2.

[Figure 2 near here]

The intended sample of those that had already had a medical examination due to tinnitus was evident as $93.15 \%$ had seen their General Practitioner and $70.55 \%$ reported having seen and Ear Nose and Throat specialist. It was also found that the greater part were underserved with tinnitus intervention as $77.5 \%$ had not had access to previous interventions. Of those that had had treatment, this included, Audiological treatment (13.70\%), tinnitus retraining therapy $(2.05 \%)$ medical interventions (4.11\%), Psychological treatments $(2.05 \%)$, complementary therapies (1.37\%). The majority (89.04\%) were not attending tinnitus support groups and therefore not receiving additional tinnitus support. The reach, therefore, included adults across the UK who previously had no access to services that provided management strategies for their tinnitus.

The context of the RCT participants

The social, demographical and socio-economic characteristics of the 146 participants who participated in the trial are shown in Table 2. There were no significant differences for any demographical or clinical variables between the experimental and control groups at baseline that may have created bias.

\section{[Table 2 near here]}

A good range of ages were represented in the study (24-83 years) with a mean of 55.57 years (SD: 12.86). The mean TFI score for tinnitus severity was 59.49 (SD: 18.40) with a range of 
28.40 to 96.40 . There were $21(12.43 \%)$ participants excluded as their TFI scores were below the inclusion level of 25 (Meikle et al., 2012).

The mean anxiety score on the GAD-7 was 7.39 (SD: 5.56) indicating mild anxiety. The majority or participants had either minimal $(36.30 \%)$ or mild anxiety $(32.88 \%)$. There were fewer participants with moderate (19.18\%) or severe anxiety (11.64\%). A similar pattern was found for the mean depression scores on the PHQ-9 (Spitzer et al., 1999) with a score of 7.99 (SD: 5.72$)$. The majority had mild depression (67.12\%). The ISI indicated that the majority of participants had subthreshold insomnia $(32.19 \%)$ or moderate insomnia $(28.77 \%)$. Selfreported hearing disability indicated that the majority $(43.84 \%)$ had $50 \%$ probability of hearing disability, with $36.99 \%$ using hearing aids.

Participants from a large range of educational and vocational backgrounds were drawn to the study. This included $56.53 \%$ who were working and $43.47 \%$ were retired or unemployed. A range of professions were drawn to the study, including those with technical, service, sales, and administrative roles. Education levels also ranged widely with around half educated to high school or college level and the other half having received vocational or degree training.

Participants were asked to rate how committed they were to the programme on a scale of 1-10 at the start of the intervention. The mean rating was 9.39 (SD: 1.10). To fully benefit, participants had to be willing to try new techniques to learn to minimise the impact of tinnitus. Before starting they were asked to rate how willing they were to try new things on a scale of 1-10. The mean rating was 9.51 (SD: 1.06). They were therefore very motivated and willing to try new techniques before starting the programme. 
The context of the research included a wide range of demographic backgrounds. Participants also presented with varying severity levels for both tinnitus distress and for the associated difficulties such as anxiety, depression, and insomnia. They appeared committed and willing to try the intervention prior to commencing.

\section{Processes related to the intervention delivery:}

Amount of treatment (dose) delivered

The intervention modules were released on a weekly basis over an eight-week period. Each week two recommended modules were released. An additional weekly optional module was released between weeks 3-7. Participants were instructed to engage with the modules and then practise the suggested techniques on a daily basis. To ensure that the intervention was accessible the information could be read online, downloaded to read offline or printed. It was also accessible in a range of formats including computers (both PC and Mac), tablets and smart phones.

The intervention was guided, and the quality of the delivery would, therefore, be partly affected by the nature of this guidance. To maintain consistency with the standard approach of tinnitus therapy being delivered within the Audiology community in the UK, an Audiologist guided this intervention. An Audiological Scientist appropriately trained to Masters Level and registered with the Health and Care Professions Council (HCPC) undertook the role of supporting the participants. The Audiologist was experienced in managing tinnitus patients both in a clinical setting and online and had a suitable understanding of CBT principles but no formal CBT training. The guidance included conducting the telephone interviews, introducing weekly modules, providing feedback, answering queries, supporting and encouraging engagement. The recommended time 
Beukes. Process evaluation implementing iCBT for tinnitus in a RCT

guideline of a minimum of 10 minutes per participant was applied to this study. The clinician was alerted every time participants completed a worksheet and they were then given feedback in the form of a written message. These messages provided advice and further suggestions as required. The Audiologist sent 1,925 tailored messages (15.65 on average to each participant). The guidance was provided in an asynchronous manner using a secure encrypted messaging system. This enabled two-way communication between the Audiologist and the participants. Synchronous guidance using telephone calls was provided with a minimum of an introduction telephone call and a summary telephone call. Additional calls were made as required during the intervention to encourage engagement or to address particular difficulties an individual was having. A comprehensive, guided, evidence-based intervention was therefore delivered in an accessible format with the recommended amount of guidance.

\section{Amount of treatment (dose) received}

The majority of participants were able to utilise the intervention. For those that withdrew, reasons given were generally that they were too busy or developed poor health. The extent to which participants actively engaged and interacted with the resources provided by this iCBT intervention was used to determine the dose received. Participants logged into the programme an average of 27 times. An average of $74.33 \%$ of the recommended modules and $55.36 \%$ of the optional module were read. The optional modules and modules occurring later in the intervention were read less than the earlier modules. Overall $41.0 \%$ of the worksheets were completed. A similar trend to that seen with the module reads was observed with more worksheets completed for the initial modules and less for the later and optional modules, as seen in Figure 3. 
Participants were monitored weekly for tinnitus severity whilst the experimental group undertook the intervention $\left(\mathrm{T}_{0}-\mathrm{T}_{1}\right)$. From week 4 to 8 there were significant differences, as the experimental group's tinnitus distress was rated significantly lower than that of the control group who were not undergoing the intervention. This trend of lowering tinnitus distress for the experimental group is seen in Figure 3. Therefore, although fewer modules and worksheets were completed as the intervention progressed, this may partly be due to an improvement in tinnitus-related distress and therefore less need for the programme.

Satisfaction of the module information for each week is also seen in Figure 3. This trend was more linear and did not appear to have an impact on the number of modules read or worksheets completed.

\section{[Figures 3 near here]}

Participants sent fewer messages than the therapist at 597 messages with an average of 4.85 per participant that completed the intervention. At the end of the intervention, participants indicated that they spent an average of 22.38 (SD: 18.95) minutes per week going through the module content with ranges between 5-60 minutes reported. Some participants (17.12\%) mentioned that it was difficult to fit in the work within the eight week period. For these participants, the time period was extended by two weeks.

Despite not all participants fully interacting with the intervention, significant positive postintervention results were obtained with a between-group effect size of Cohen's $d=0.76$ for the TFI. 


\section{Processes related to the outcomes obtained:}

\section{Implementation fidelity}

This intervention was delivered as planned. There were no changes to the methods or outcome measures used after trial commencement. This was partly due to methodology followed in that initially the RCT study protocol was peer reviewed and amendments followed these recommendations. Further changes were incorporated after tinnitus professionals evaluated the programme in terms of acceptability and suitability (see Beukes et al., 2016). A pilot study moreover identified shortcomings. The intervention and protocol were adapted accordingly (see Beukes et al., 2017a). Therefore the protocol and intervention had already been modified prior to the RCT commencing. The only modification was extending the treatment period by two weeks for those that were unable to complete the intervention within the given timeframe due to unforeseen circumstances.

\section{Barriers to implementation}

Participants who completed the intervention were asked to rate the programme in order to determine where barriers to intervention usage existed. As participants who completed the intervention only gave these ratings, an element of bias was added. Overall the intervention was rated highly, with a mean score of greater than 4 out of 5 for most categories as indicated in Figure 4. The three areas with scores below 4 were self-motivation, completing the worksheets, and understandability of the intervention. These barriers can affect the application of this intervention. Self-motivation appeared to decrease for some participants as seen by the number of modules read and the worksheets completed for later parts of the intervention. Some of the worksheets with too many areas to complete (e.g. sections for the date, effectiveness rating, what was done, further comments) could be simplified. The intervention content would benefit from revision to improve understandability. 


\title{
[Figure 4 near here]
}

\author{
A further barrier encountered was motivating participants to complete the end of intervention \\ and follow-up questionnaires. Despite three automatic reminders as well as further personal \\ reminders by the Audiologist, $92.47 \%$ of the participants completed the assessment measures \\ post treatment, whilst $78.08 \%$ completed these at follow-up. Keeping some participants \\ engaged during the intervention was also challenging. Messages were sent to those who were \\ not engaging. If there was no response, a telephone call was made to them. Some participants \\ found the lack of immediate results deterred them from continuing with the intervention. \\ Self-motivation, family issues, new health problems, and time-pressures were the main \\ barriers to implementation that were identified.
}

Google Analytics indicated that the majority of those looking at the recruitment website were aged 25 to 34 years (33.54\%), and the least aged 55 to 64 years or over 65 years (both at $5.52 \%)$. Of interest is that those that chose to participate were generally older and a much smaller percentage of those aged below 35 years decided to participate. Of those that viewed the recruitment pages, $54.11 \%$ were male and $45.89 \%$ were female. A slightly higher proportion of men joined the study (56.85\%). Strategies to minimise barriers and reach populations who were hesitant to partake are required.

\section{Facilitation of effectiveness}

Following undertaking the intervention participants were asked to rate how beneficial different components within the intervention were. The results are indicated in Figure 5. The applied relaxation modules were rated the most beneficial, while the hearing tactics module 
was rated the least beneficial. These ratings were also reflected in some of the outcomes obtained. There were no significant group differences in hearing disability directly following the experimental group undertaking the intervention $F(1,145)=2.06 ; p=0.154$ (Beukes et al. 2017b). The hearing tactics module was included as an optional module to provide tactics to minimise hearing disability, however, many participants chose not to do it. Participants may not have realised the relevance of the module in the context of a tinnitus intervention. Outlining the rationale of the hearing tactics module should be incorporated in future trials.

\begin{abstract}
Although the sleep guidelines were not rated as high as some of the other modules there was a significant positive between-group difference (Cohen's $d=0.55$ ) after the experimental group completed the intervention $\left(\mathrm{T}_{0}-\mathrm{T}_{1}\right.$; Beukes et al. 2017b). The interaction of the various modules may have contributed to improvements seen post-intervention on assessment measures for insomnia, depression, and quality of life.
\end{abstract}

\title{
[Figure 5 near here]
}

From the responses to open-ended questions, it was evident that an Internet-intervention was valued by participants due to the ability to access help in the comfort of their own homes, without needing to be away from work for a hospital appointment. Furthermore, working at their own pace was an advantage. Participants also found it useful to access the information when they were out, such as on a train, due to the website being able to be viewed on mobile devices as well due to the responsive nature of the website design. These factors contributed to the effectiveness of the intervention.

\section{Discussion}

Process evaluation was performed to identify aspects that could facilitate or hinder clinical implementation of an Internet-based cognitive behavioural therapy intervention for tinnitus 
Beukes. Process evaluation implementing iCBT for tinnitus in a RCT

(iCBT) in the UK. This was done by exploring the research context, the intervention components and the factors that contributed to the outcomes obtained.

\section{The impact of the research context}

The recruitment strategy was expanded following the pilot study and was effective at recruiting the required sample size. Some people who registered for the trial did not pursue the intervention. This was related to the timing of the invention not being convenient in most cases. Considering the high prevalence of tinnitus, recruitment strategies will need to be expanded if more individuals are to be made aware of this intervention.

The intended sample of those with distressing tinnitus who were underserved with evidencebased tinnitus interventions was reached. The majority had not had previous tinnitus treatments and were not attending tinnitus support groups. A large proportion of these $(70.55 \%)$ indicated that they had seen an ENT specialist. This percentage is higher than the estimated referral rates to specialist services of $37 \%$ by general practitioners (El-Shunner et al., 2011). Whilst definitive information about what would be an optimal rate of referral to secondary care for tinnitus is not available, there is evidence of an unmet need in the tinnitus population (Gander et al. 2011). Of those that had accessed previous interventions, $13.70 \%$ indicated they had had previous audiological treatments. This is in line with current estimated referral rates of $12 \%$ by General Practitioners (El-Shunner et al., 2011). Few had had psychological treatments which generally cover CBT principles. The cognitive behavioural therapy aspect of the intervention may, therefore, have drawn some participants.

The demographical spread of participants was UK wide, although fewer participants were from the regions, such as from Scotland or Wales. This could partly reflect the effect of the 
Beukes. Process evaluation implementing iCBT for tinnitus in a RCT

variation of availability of clinical tinnitus provision in the UK (Hoare et al. 2015). Strategies to improve the spread in areas not reached needs consideration.

The context of this research indicated that iCBT was applicable for adults of different ages and educational background. It also encompassed those with varying levels of tinnitus severity and duration of having tinnitus.

\section{The impact of the intervention delivery}

A comprehensive Audiology-guided intervention was delivered. A guided intervention was selected as therapeutic support can improve engagement with Internet interventions (Kohl, Crutzen \& de Vries, 2013). Guidance was provided by Clinical Psychologists during previous European iCBT studies. They were either licensed CBT therapists, Clinical Psychology (Master' degree), or MSc students who had completed their clinical and CBT training. These therapists received systematic training and supervision to guide iCBT interventions. Previous studies had a lower therapist-participant ratio, as more than one therapist guided the intervention [i.e. six therapists by Hesser et al. (2012), four therapists by Weise et al. (2016) and Jasper et al. (2014); three therapists by Kaldo et al. (2008)]. Although these therapists had a good understanding of CBT they had not always had previous experience with tinnitus patients. There were, therefore, large differences related to the therapist in this study compared to those in previous studies in terms of training and experience. Having one therapist, as in this trial, could produce a consistent approach for all participants.

Despite these therapeutic differences, outcomes of this trial on tinnitus distress (Cohen's $d=$ 0.69) were comparable to those of the previous iCBT controlled studies with pooled results of Hedges $g=0.58$ (Andersson, 2015). The exact influence of the health professional assigned is not yet known. Internet-based trials for depression, anxiety, and social phobia have found

\footnotetext{
E-mail: editor-ija@utdallas.edu URL: http://mc.manuscriptcentral.com/tija
} 
comparable results, regardless of whether a clinician or a technical assistant guided the intervention (Titov et al. 2009; Robinson et al. 2010; Titov et al. 2010). Outcomes obtained may be related to numerous factors and not only based on the qualification of the person guiding the intervention. An Audiologist may not be as experienced in motivational techniques required to improve engagement and attrition. Direct comparison of Audiologydelivered versus Psychology-delivered interventions is required to draw firm conclusions. Although participants on average did not utilise the full scope of both the core and optional aspects of the intervention, undertaking the intervention led to a significant reduction in tinnitus and problems associated with tinnitus based on the results from our outcome data. Additional tactics to improve engagement need to be considered.

\section{Processes related to the outcomes obtained}

Implementation fidelity was high. Preceding this trial with a feasibility study assisted as barriers could be addressed prior to running the RCT. Participants indicated great satisfaction with, and benefit from, the intervention. The ease of intervention access was an advantage as well as being able to learn more about managing their tinnitus. Although patients who access tinnitus services are well supported, there are many who do not have access to these services (Hoare et al., 2015). Implementation of iCBT requires fewer resources due to the intervention being provided via the Internet. Due to these benefits, it has been incorporated into regular clinical care in Sweden (Kaldo-Sandström, 2004; Kaldo et al. 2013). Adding iCBT as an additional treatment pathway in the UK could increase access to a comprehensive evidence-based intervention for those with distressing tinnitus.

Younger adults and females were less inclined to participate. Gender differences may be related to the estimates that a slightly higher proportion of men experience tinnitus, although 
a higher proportion of women have a severe form of tinnitus (Seydel et al., 2013). It could also be that the flexibility and anonymous nature of this intervention suited males. Addressing factors deterring certain recipients, from participating is important.

Self-motivation is a requirement for such a self-help intervention. Ways of improving motivation are required. Improvements to aspects of the intervention that were poorly rated, such as simplifying the worksheets is required. Time-restrictions and poor-health resulted in not all participants being fully engaged with the intervention. Developing a more tailored intervention with key principles is something that may be considered to target those who are unable to commit to an eight-week programme. It was found that over time engagement decreased but so did tinnitus severity. Improved tinnitus severity may have led to less reliance on the intervention over time. Some participants also mentioned finding the lack of initial results difficult, which decreased motivation. Tinnitus distress was significantly lower after completing 4-weeks of the intervention (Beukes et al. 2017b). These findings should be explained to future participants as both encouragement and to help adjust their expectations.

\section{Further research}

Additional longitudinal analysis of the patient journey would be of value. Qualitative analysis of participant expectation and experiences (both positive and negative) are required. This could provide valuable insights into factors that could deter participants from undertaking the intervention. Evaluating the patient journey from the perspective of their significant others would add further insight into the application of iCBT. 


\section{Conclusions}

This process evaluation has provided a broader understanding of the research context. Aspects that contributed to the effectiveness of the intervention were identified. Barriers restricting engagement such as time-limitations and low motivation levels need addressing. Results of this process evaluation together with the outcome data can be used to facilitate translating this research into clinical practice.

\section{Funding and disclosure statement}

The authors declare that they have no competing interests. Anglia Ruskin, Lamar and Linköping Universities and the National Institute for Health Research supported the undertaking of this study but the views expressed are those of the authors and not of these institutions.

\section{References}

Abbott, J., M, Kaldo, V., Klein, B., Austin, D., Hamilton, C., et al. A cluster randomised controlled trial of an Internet-based intervention program for tinnitus distress in an industrial setting. Cog Behav Ther 2009; 38: 162-173.

Andersson, G. Clinician-supported Internet-delivered psychological treatment of tinnitus. Am J Audiol 2015; 24(3): 299-30.

Andersson, G., Kaldo, V. Cognitive-behavioral therapy with applied relaxation. In R. S. Tyler (Ed.), Tinnitus treatment. Clinical protocols. New York: Thieme 2006: 96-115. Andersson, G., Kaldo-Sandström, V. Treating tinnitus via the Internet. CME Journal. Otorhinolaryngol, Head Neck Surg 2003; 7: 38-40.

Andersson, G., Strömgren, T., Ström, T., Lyttkens, L. Randomised controlled trial of Internet based cognitive behavior therapy for distress associated with tinnitus. 
Beukes. Process evaluation implementing iCBT for tinnitus in a RCT

Psychosom Med 2002, 64: 810-816.

Baranowski, T., Stables, G. Process evaluations of the 5-a-day projects, Health education \& behavior: the official publication of the Society for Public Health Education 2000; 27(2): 157-166.

Bastien, C.H., Vallières, A.; Morin, C.M. Validation of the Insomnia Severity Index as an outcome measure for insomnia research. Sleep medicine 2001, 2(4): 297-307.

Beukes, E.W., Manchaiah, V., Allen, P.M., Baguley, D.M., Andersson, G. Internet-based cognitive behavioural therapy for adults with tinnitus in the UK: study protocol for a randomised controlled trial, BMJ open 2015; 5(9). e008241-2015-008241.

Beukes, E.W., Vlaescu, G., Manchaiah, V., Baguley, D.M., Allen, Andersson, G. Development and technical functionality of an Internet-based intervention for tinnitus in the UK, Internet Interv 2016; 6: 6-15.

Beukes, EW. Allen, PM, Manchaiah, V., Baguley, DM., Andersson, G. Internet-based intervention for tinnitus: Outcome of a single-group open trial. J Am Acad Audiol 2017a; $00,1-12$.

Beukes, E.W., Baguley, D.M., Manchaiah, V., Allen, P.M., Andersson, G. Audiology-Guided Internet-delivered Cognitive Behaviour Therapy for Adults with Tinnitus in the UK. JHS 2017b; 7(2): 92 .

Beukes, E.W., Baguley, D.M., Allen, P.M., Manchaiah, V. and Andersson, G. Guided Internet-based versus face-to-face clinical care in the management of tinnitus: study protocol for a multi-centre randomised controlled trial. Trials 2017c; 18(1): 186.

Craig, P., Dieppe, P., Macintyre, S., Michie, S., Nazareth, I., et al. Medical Research Council Guidance, Developing and evaluating complex interventions: the new Medical Research Council guidance", BMJ (Clinical research ed.) 2008; 337, a1655.

Davis, A., Rafaie, E.A. Epidemiology of tinnitus, Tinnitus handbook 2000; 1-23. 
Beukes. Process evaluation implementing iCBT for tinnitus in a RCT

Dzewaltowski, D.A., Glasgow, R.E., Klesges, L.M., Estabrooks, P.A., Brock, E. RE-AIM: evidence-based standards and a Web resource to improve translation of research into practice. Ann Beh Med 2004; 28(2): 75-80.

El-Shunnar, S.K., Hoare, D.J., Smith, S., Gander, P.E., Kang, S., et al. Primary care for tinnitus: practice and opinion among GPs in England, J Eval Clin Pract 2011; 7(4): 684692.

Gander, P.E., Hoare, D.J., Collins, L., Smith, S., Hall, D.A. Tinnitus referral pathways within the National Health Service in England: a survey of their perceived effectiveness among audiology staff, BMC Health Serv Res 2011; (1)1.

Glasgow, R.E., Vogt, T.M. \& Boles, S.M. 1999, Evaluating the public health impact of health promotion interventions: the RE-AIM framework, Am J Public Health 2011; 89 (9): $1322-1327$.

Grant, A., Treweek, S., Dreischulte, T., Foy, R., Guthrie, B. Process evaluations for clusterrandomised trials of complex interventions: a proposed framework for design and reporting. Trials 2013; 14(1): 15.

Grewal, R., Spielmann, P., Jones, S., Hussain, S. Clinical efficacy of tinnitus retraining therapy and cognitive behavioural therapy in the treatment of subjective tinnitus: a systematic review, J Laryngol Otol 2014; 128 (12): 1028-1033.

Grimshaw, J.M., Eccles, M.P., Lavis, J.N., Hill, S.J., Squires, J.E. Knowledge translation of research findings, Implement Sci 2012; 7(1): 1 .

Grol, R. Successes and failures in the implementation of evidence-based guidelines for clinical practice, Med care 2001; 39 (8): 11-46.

Gussenhoven, A.H.M., Singh, A.S., Goverts, S.T., van Til, M., Anema, J.R. et al. A process evaluation of implementing a vocational enablement protocol for employees with hearing difficulties in clinical practice. Int J Audiol 2015; 54(8), 507-517. 
Beukes. Process evaluation implementing iCBT for tinnitus in a RCT

Hesser, H., Weise, C., Westin, V.Z., Andersson, G. A systematic review and meta-analysis of randomized controlled trials of cognitive-behavioral therapy for tinnitus distress, Clin Psychol Rev 2011; 31(4): 545-553.

Hesser, H., Gustafsson, T., Lundén, C., Henrikson, O., Fattahi, K., Johnsson, E., Westin, V.Z., Carlbring, P., Mäki-Torkko, E., Kaldo, V., Andersson, G. A randomized controlled trial of internet-delivered cognitive behavior therapy and acceptance and commitment therapy in the treatment of tinnitus. J. Consult. Clin. Psychol 2012; 80(4): 649.

Hoare, D.J., Broomhead, E., Stockdale, D., Kennedy, V. Equity and person-centeredness in provision of tinnitus services in UK National Health Service audiology departments, EJPCH 2015; 3(3): 318-326.

Jasper, K., Weise, C., Conrad, I., Andersson, G., Hiller, W., et al. Internet- based guided selfhelp versus group cognitive behavioral therapy for chronic tinnitus: a randomized controlled trial, J Psychosom Res 2014; 83 (4), 234-246.

Kaldo, V., Cars, S., Rahnert, M., Larsen, H.C., Andersson, G. Use of a self-help book with weekly therapist contact to reduce tinnitus distress: a randomized controlled trial, $J$ Psychosom Res 2007; 63 (2): 195-202.

Kaldo, V., Levin, S., Widarsson, J., Buhrman, M., Larsen, H.C., Andersson, G. Internet versus group cognitive-behavioral treatment of distress associated with tinnitus: a randomized controlled trial. Behav Ther 2008; 39(4):.348-359.

Kaldo, V., Haak, T., Buhrman, M., Alfonsson, S., Larsen, H. et al. Internet-based cognitive behaviour therapy for tinnitus patients delivered in a regular clinical setting: Outcome and analysis of treatment dropout, Cogn Behav Ther 2013; 42(2): 146-158.

Kaldo-Sandström, V., Larsen, H.C., Andersson, G. Internet- based cognitive-behavioral selfhelp treatment of tinnitus: Clinical effectiveness and predictors of outcome, Am J Audiol 2004; 13(2): 185-192. 
Kohl, L.F., Crutzen, R., de Vries, N.K. Online prevention aimed at lifestyle behaviors: a systematic review of reviews, J Med Int Res 2013; 15(7): e146.

Landgrebe, M., Azevedo, A., Baguley, D., Bauer, C., Cacace, A., et al. Methodological aspects of clinical trials in tinnitus: a proposal for an international standard, $J$ Psychosom Res 2012; 73(2): 112-121.

Linnan, L., Steckler. A. Process evaluation for public health interventions and research (pp. 1-23). San Francisco: Jossey-Bass 2002.

Manchaiah, V., Danermark, B., Rönnberg, J., Lunner, T. Importance of process evaluation in audiological rehabilitation: Examples from studies on hearing impairment. Int J Otol 2014, Article ID 168684. http://dx.doi.org/10.1155/2014/168684.

Martinez, C., Wallenhorst, C., McFerran, D., Hall, D.A. Incidence rates of clinically significant tinnitus: 10-year trend from a cohort study in England, Ear Hear 2015; 36(3): e69-75.

Meikle, M.B., Henry, J.A., Griest, S.E., Stewart, B.J., Abrams, H.B., et al. 2012. The tinnitus functional index: development of a new clinical measure for chronic, intrusive tinnitus, Ear Hear 2012; 33(2), 153-176.

Moore, G.F., Audrey, S., Barker, M., Bond, L., Bonell, C., Hardeman, W., Moore, L., O’Cathain, A., Tinati, T., Wight, D. and Baird, J. Process evaluation of complex interventions: Medical Research Council guidance. BMJ 2015; 350: p.h1258.

Newman, C.W., Sandridge, S.A., Bolek, L. Development and psychometric adequacy of the screening version of the tinnitus handicap inventory, Otol Neurotol 2008; 29(3), 276281.

Parliament, B. Data protection act of 1998.

Riach, E., The Privacy and Electronic Communications Directive. New Law Journal 2003: 1(7071):.379-380. 
Beukes. Process evaluation implementing iCBT for tinnitus in a RCT

Robinson, E., Titov, N., Andrews, G., McIntyre, K., Schwencke, G. and Solley, K. Internet treatment for generalized anxiety disorder: a randomized controlled trial comparing clinician vs. technician assistance. PloS one 2010; 5(6), p.e10942.

Saunders, R.P., Evans, M.H., Joshi, P. Developing a process-evaluation plan for assessing health promotion program implementation: a how-to guide, Health Promot Pract 2005; 6(2): 134-147.

Seydel, C., Haupt, H., Olze, H., Szczepek, A. J., Mazurek, B. Gender and chronic tinnitus: differences in tinnitus-related distress depend on age and duration of tinnitus. Ear Hear 2013; 34(5): 661-672.

Spitzer, R.L., Williams, J.B. and Kroenke, K. Patient Health Questionnaire: PHQ. New York State Psychiatric Institute 1999.

Spitzer, R.L., Kroenke, K., Williams, J.B. and Löwe, B., 2006. A brief measure for assessing generalized anxiety disorder: the GAD-7. Arch Intern Med 2006; 166(10): 1092-1097.

Steckler, A.B., Linnan, L. Israel, B. Process evaluation for public health interventions and research, Jossey-Bass San Francisco, CA 2002.

Sung, N.S., Crowley Jr, W.F., Genel, M., Salber, P., Sandy, L., et al. 2003. Central challenges facing the national clinical research enterprise, Jama 2003; 289(10): 1278-1287.

Titov, N., Andrews, G., Davies, M., McIntyre, K., Robinson, E., Solley, K. Internet treatment for depression: a randomized controlled trial comparing clinician vs. technician assistance. PLoS One 2010; 5, e10939. doi:10.1371/journal.pone.0010939.

Titov, N., Andrews, G., Schwencke, G., Solley, K., Johnston, L., Robinson, E. An RCT comparing effect of two types of support on severity of symptoms for people completing Internet-based cognitive behaviour therapy for social phobia. Aust $N Z$ J Psychiatry 2009; 1(43), 920-926.

E-mail: editor-ija@utdallas.edu URL: http://mc.manuscriptcentral.com/tija 
Wanless, D. Securing good health for the whole population, London: HM Treasury 2004; 4: 2003-2004.

Weise, C., Kleinstauber, M., Andersson, G. Internet-Delivered Cognitive-Behavior Therapy for Tinnitus: A Randomized Controlled Trial, Psychosom Med 2016; 78(4), 501-510. 
Beukes. Process evaluation implementing iCBT for tinnitus in a RCT

\section{Figure Legends}

Figure 1: Participant profile

Figure 2: The demographic location of the participants

Figure 3: Comparison of engagement, module rating and weekly tinnitus distress

Figure 4: Participation ratings of the intervention

Figure 5: Usefulness of specific modules within the intervention (optional modules marked with a *) 
Table 1: An overview of the iCBT intervention

\begin{tabular}{|c|c|c|c|c|}
\hline \multirow{2}{*}{$\begin{array}{l}\text { Time } \\
\text { line }\end{array}$} & \multirow[t]{2}{*}{ Intervention content } & \multirow{2}{*}{$\begin{array}{l}\text { Short } \\
\text { worksheets } \\
\text { or quizzes }\end{array}$} & \multicolumn{2}{|c|}{ Intervention load } \\
\hline & & & $\begin{array}{l}\text { Weekly } \\
\text { Reading }\end{array}$ & $\begin{array}{l}\text { Daily } \\
\text { practising }\end{array}$ \\
\hline $\begin{array}{l}\text { Week } \\
1\end{array}$ & $\begin{array}{l}\text { Programme rational and outline } \\
\text { Understanding tinnitus }\end{array}$ & $\begin{array}{l}3 \\
5\end{array}$ & $\begin{array}{l}15 \text { minutes } \\
15 \text { minutes }\end{array}$ & \\
\hline $\begin{array}{l}\text { Week } \\
2\end{array}$ & $\begin{array}{l}\text { Deep relaxation } \\
\text { Positive imagery } \\
\text { Sound enrichment* }\end{array}$ & $\begin{array}{l}2 \\
2 \\
3\end{array}$ & $\begin{array}{l}10 \text { minutes } \\
10 \text { minutes } \\
10 \text { minutes }\end{array}$ & $\begin{array}{l}10 \text { minutes } \\
5 \text { minutes } \\
\text { As required }\end{array}$ \\
\hline $\begin{array}{l}\text { Week } \\
3\end{array}$ & $\begin{array}{l}\text { Diaphragmatic breathing } \\
\text { Reinterpreting tinnitus } \\
\text { Sleep management* }\end{array}$ & $\begin{array}{l}4 \\
5 \\
6\end{array}$ & $\begin{array}{l}10 \text { minutes } \\
10 \text { minutes } \\
15 \text { minutes }\end{array}$ & $\begin{array}{l}10 \text { minutes } \\
5 \text { minutes } \\
\text { As required }\end{array}$ \\
\hline $\begin{array}{l}\text { Week } \\
4\end{array}$ & $\begin{array}{l}\text { Entire body relaxation } \\
\text { Focusing techniques } \\
\text { Concentration management* }\end{array}$ & $\begin{array}{l}2 \\
2 \\
7\end{array}$ & $\begin{array}{l}10 \text { minutes } \\
10 \text { minutes } \\
10 \text { minutes }\end{array}$ & $\begin{array}{l}5 \text { minutes } \\
5 \text { minutes } \\
\text { As required }\end{array}$ \\
\hline $\begin{array}{l}\text { Week } \\
5\end{array}$ & $\begin{array}{l}\text { Rapid relaxation } \\
\text { Thought analysis } \\
\text { Reducing sound sensitivity* }\end{array}$ & $\begin{array}{l}1 \\
3 \\
7\end{array}$ & $\begin{array}{l}10 \text { minutes } \\
15 \text { minutes } \\
15 \text { minutes }\end{array}$ & $\begin{array}{l}3 \text { minutes } \\
3 \times 15 \text { minutes } \\
\text { Daily }\end{array}$ \\
\hline $\begin{array}{l}\text { Week } \\
6\end{array}$ & $\begin{array}{l}\text { Relaxation in daily routines } \\
\text { Cognitive restructuring } \\
\text { Communication tactics* }\end{array}$ & $\begin{array}{l}1 \\
1 \\
5\end{array}$ & $\begin{array}{l}10 \text { minutes } \\
15 \text { minutes } \\
15 \text { minutes }\end{array}$ & $\begin{array}{l}3-5 \text { situations } \\
3 \times 15 \text { minutes } \\
\text { As required }\end{array}$ \\
\hline $\begin{array}{l}\text { Week } \\
7\end{array}$ & $\begin{array}{l}\text { Relaxation in stressful } \\
\text { situations } \\
\text { Exposure to tinnitus }\end{array}$ & 2 & $\begin{array}{l}10 \text { minutes } \\
10 \text { minutes }\end{array}$ & $\begin{array}{l}\text { As required } \\
3 \times 5 \text { minutes }\end{array}$ \\
\hline
\end{tabular}

E-mail: editor-ija@utdallas.edu URL: http://mc.manuscriptcentral.com/tija 
1

2

3

4

5

6

7

8

9

10

11

12

13

14

15

16

17

18

19

20

21

22

23

24

25

26

27

28

29

30

31

32

33

34

35

36

37

38

39

40

41

42

43

44

45

46

47

48

49

50

51

52

53

54

55

56

57

58

59

60

\begin{tabular}{|l|l|l|l|l|}
\hline Week & Reviewing helpful techniques & $8-13$ & 20 minutes & Evaluation \\
& $\begin{array}{l}\text { Maintenance and relapse } \\
\text { prevention }\end{array}$ & 5 & 20 minutes & Future plan \\
\hline
\end{tabular}

Optional modules to be done if required are marked with an * 


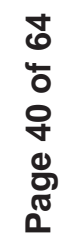

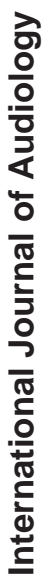

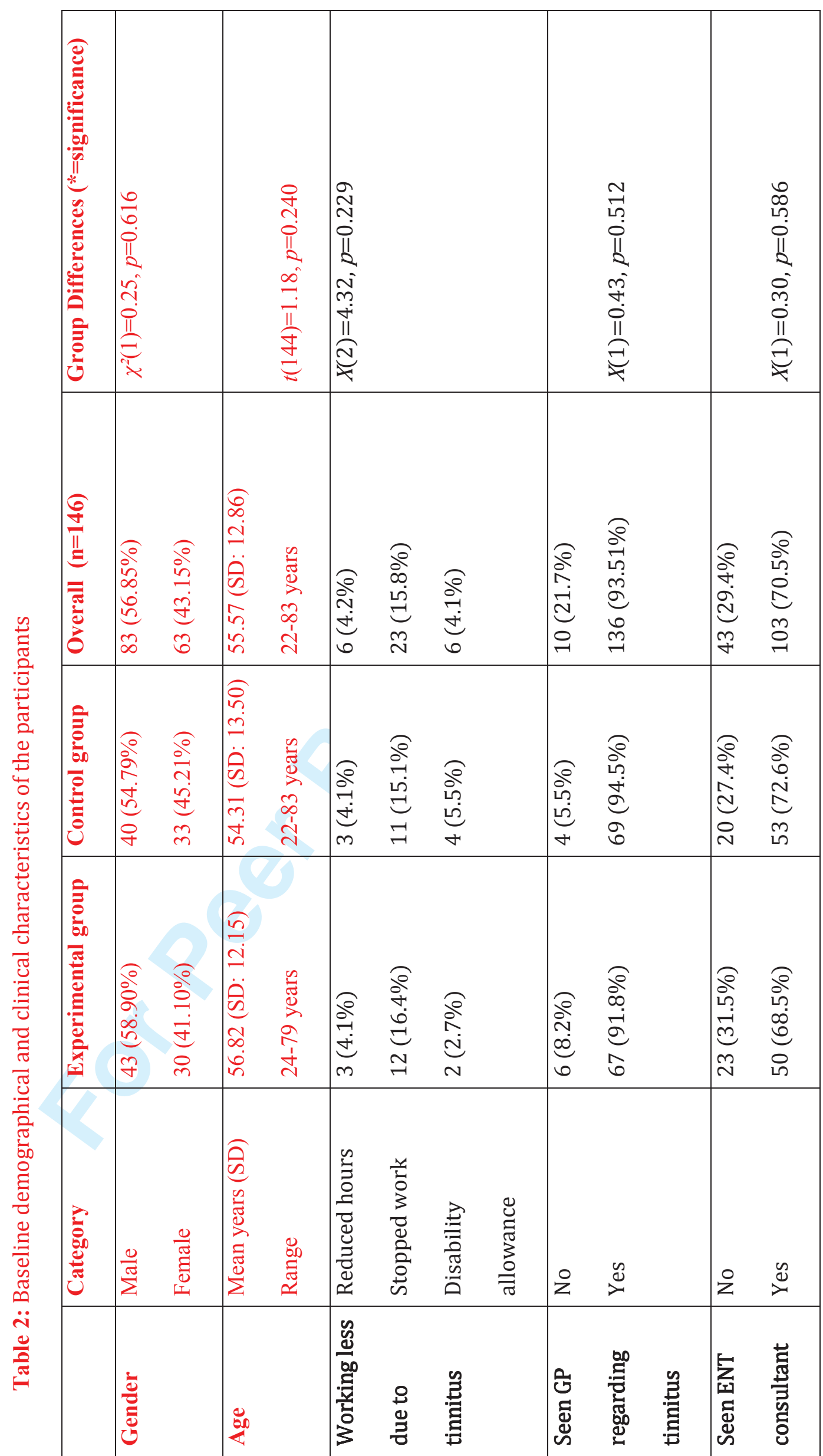




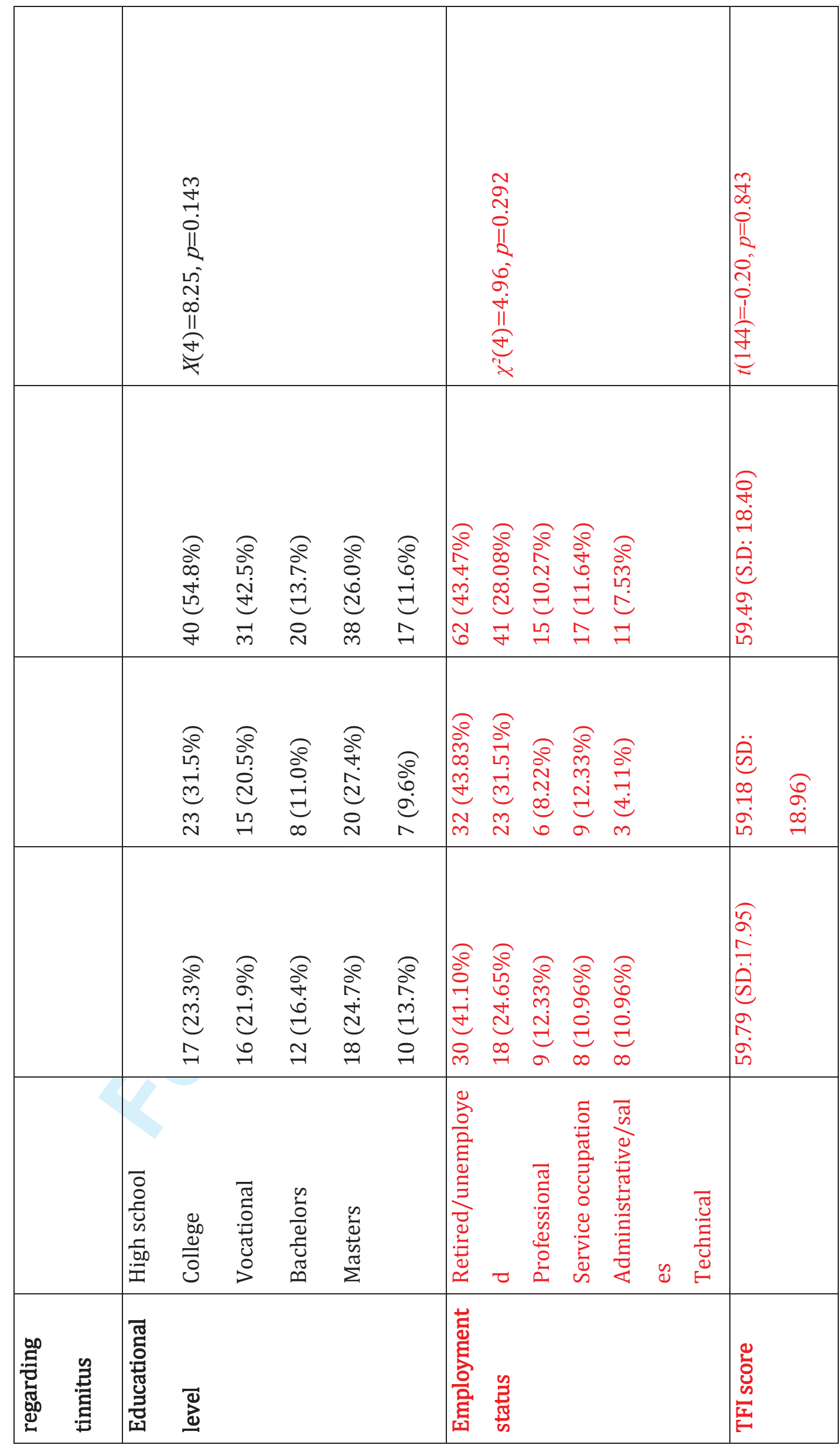


- 


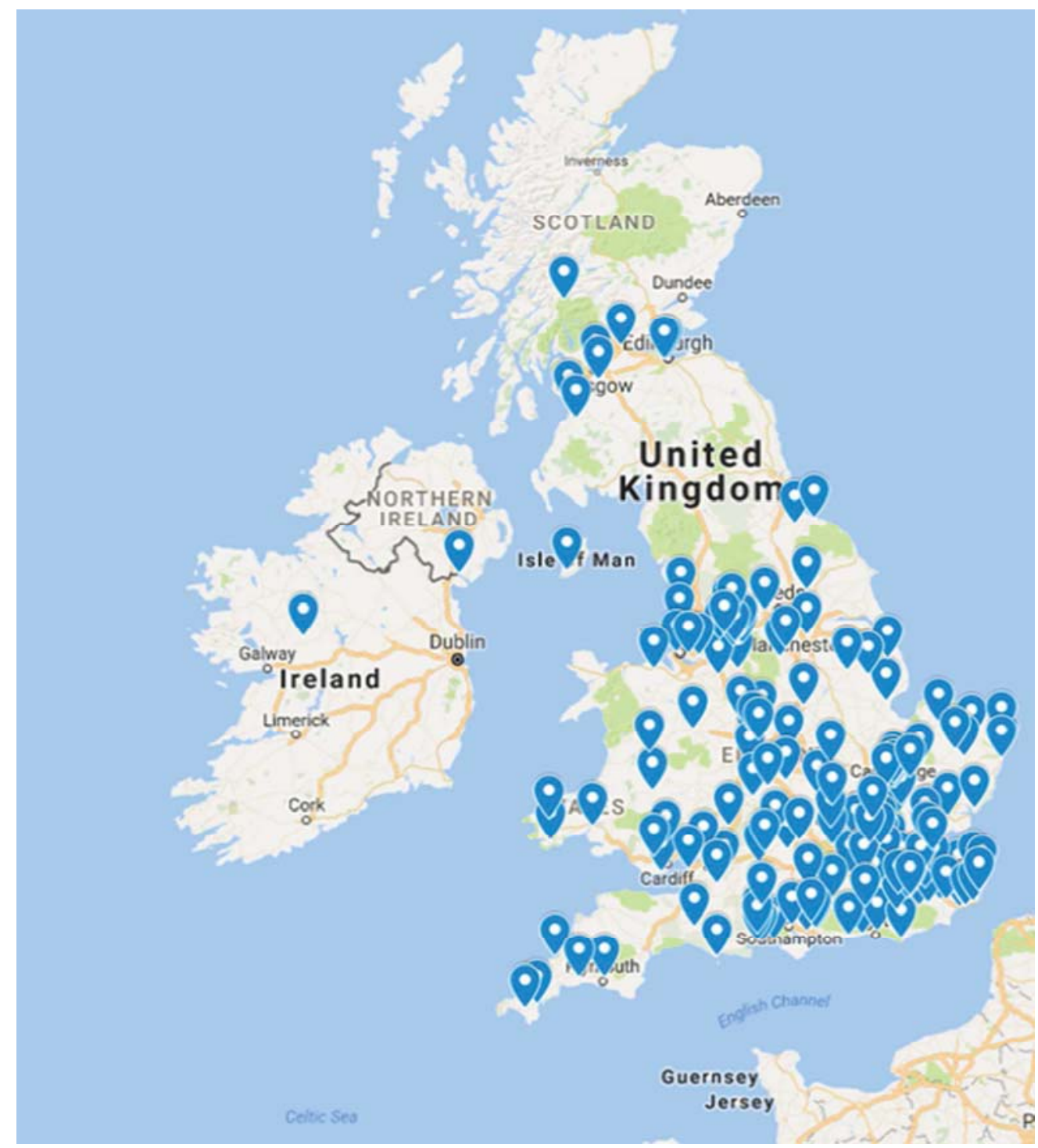

Figure 2: The demographic location of the participants $158 \times 176 \mathrm{~mm}(96 \times 96$ DPI $)$ 


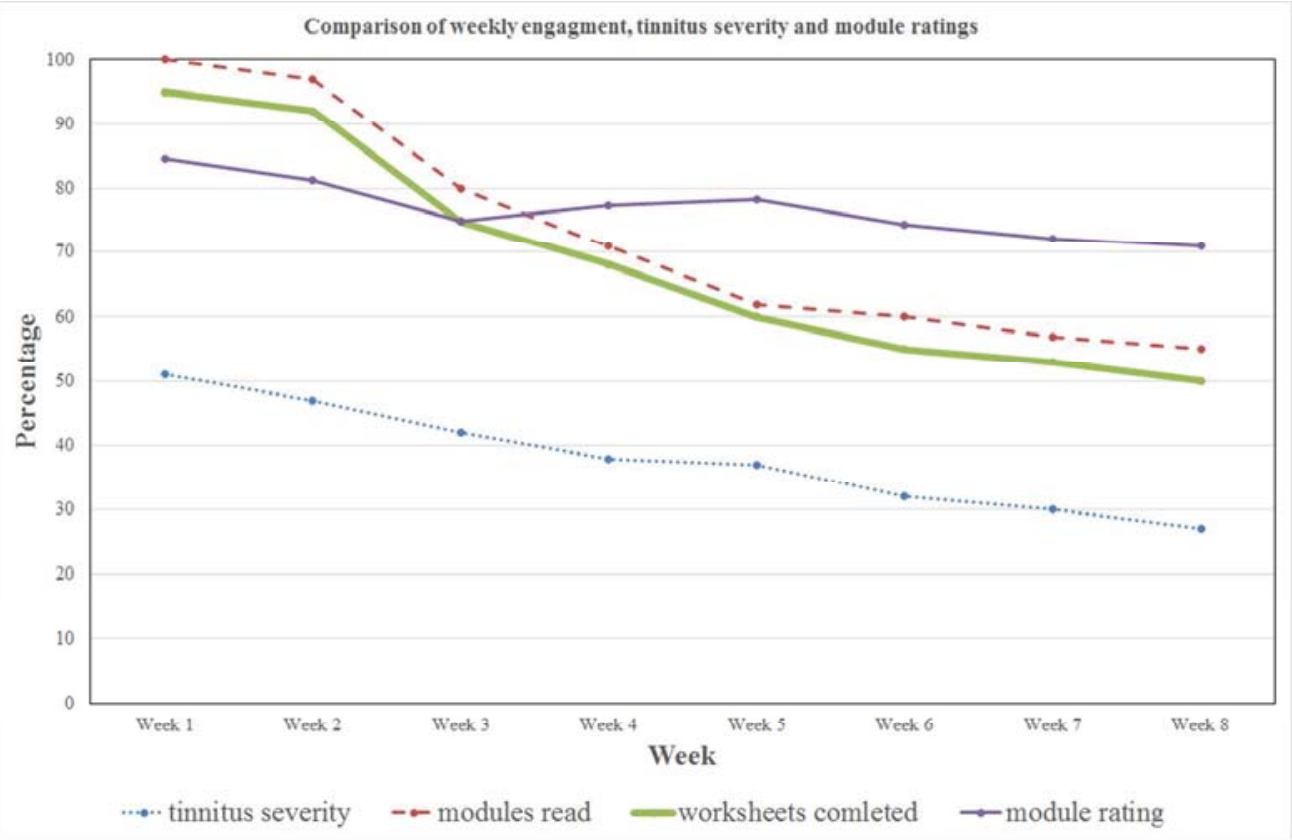

Figure 3: Comparison of engagement, module rating and weekly tinnitus distress

$$
290 \times 187 \mathrm{~mm}(96 \times 96 \mathrm{DPI})
$$




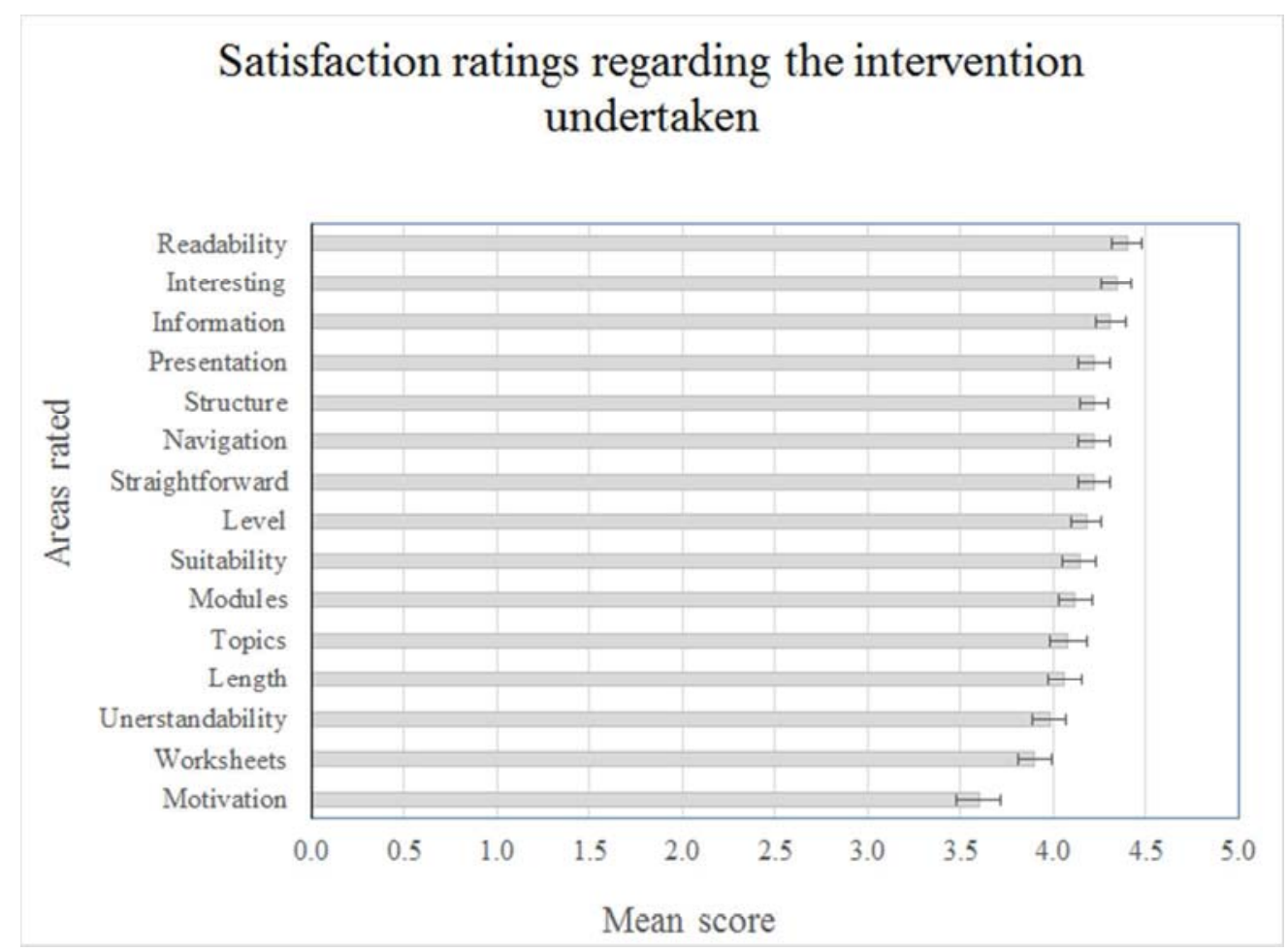

Figure 4: Participation ratings of the intervention

$189 \times 139 \mathrm{~mm}(96 \times 96 \mathrm{DPI})$

E-mail: editor-ija@utdallas.edu URL: http://mc.manuscriptcentral.com/tija 